\title{
Utility and Impact of the Implementation of Same-Day, Self-administered Electronic Patient-Reported Outcomes Assessments in Routine HIV Care in two North American Clinics
}

\author{
Duncan Short ${ }^{1}$ (D) Rob J. Fredericksen ${ }^{2} \cdot$ Heidi M. Crane ${ }^{2} \cdot$ Emma Fitzsimmons $^{2} \cdot$ Shivali Suri $^{3} \cdot$ Jean Bacon $^{4}$. \\ Alexandra Musten ${ }^{4} \cdot$ Kevin Gough $^{3} \cdot$ Moti Ramgopal $^{5} \cdot$ Jeff Berry ${ }^{6} \cdot$ Justin McReynolds $^{2} \cdot$ Abigail Kroch $^{4}$. \\ Brenda Jacobs $^{5}$. Vince Hodge ${ }^{5}$. Divya Korlipara ${ }^{5} \cdot$ William Lober $^{2}$
}

Accepted: 9 January 2022 / Published online: 22 January 2022

(C) The Author(s) 2022

\begin{abstract}
The PROgress study assessed the value and feasibility of implementing web-based patient-reported outcomes assessments (PROs) within routine HIV care at two North American outpatient clinics. People with HIV (PWH) completed PROs on a tablet computer in clinic before their routine care visit. Data collection included PROs from 1632 unique PWH, 596 chart reviews, 200 patient questionnaires, and 16 provider/staff questionnaires. During an initial setup phase involving 200 patients, PRO results were not delivered to providers; for all subsequent patients, providers received PRO results before the consultation. Chart review demonstrated that delivery of PRO results to providers improved patient-provider communication and increased the number of complex health and behavioral issues identified, recorded, and acted on, including suicidal ideation ( $88 \%$ with vs $38 \%$ without PRO feedback) and anxiety (54\% with vs $24 \%$ without PRO feedback). In post-visit questionnaires, PWH (82\%) and providers (82\%) indicated that the PRO added value to the visit.
\end{abstract}

Keywords Quality of life $\cdot$ Implementation science $\cdot$ Patient-reported outcomes $\cdot$ Suicidal ideation $\cdot$ HIV care

\section{Introduction}

Life expectancy of people with HIV (PWH) has significantly increased over the past 30 years with continuous improvements in antiretroviral therapy, in many regions reaching lengths comparable to the general population [1-3]. This increase has led to a greater focus on overall patient wellbeing and health-related quality of life as treatment outcome targets [3-5]. Focusing on person-centered healthcare

Duncan Short

duncan.x.short@viivhealthcare.com

1 Global Implementation Science, ViiV Healthcare, 980 Great West Road, Brentford, Middlesex TW8 9GS, UK

2 Center for AIDS Research, University of Washington, Seattle, WA, USA

3 St Michael's Hospital, Toronto, ON, Canada

4 Ontario HIV Treatment Network, Toronto, ON, Canada

5 Midway Specialty Care Center, Fort Pierce, FL, USA

6 TPAN, Chicago, IL, USA to address a range of determinants of poor health beyond viral suppression will enable PWH to benefit from healthy aging along with ongoing viral suppression [4]. Many symptoms, health behaviors, and life circumstances associated with living with HIV cannot be measured by laboratory values or other direct observation approaches and are often under-addressed in clinical care [6]. Rates of substance use, depression, intimate partner violence, and homelessness are higher among PWH than the general population [7-12], and a better understanding of such issues, as well as other information beyond standard clinical measures, has the potential to improve outcomes for PWH. For example, provider attempts to support PWH to maintain adherence to a treatment regimen can benefit from understanding patient behaviors and risks to adherence including substance use, depression, stigma, life circumstances such as housing status, and treatment satisfaction [13]. Furthermore, knowledge of sexual risk behavior can help identify appropriate opportunities to discuss pre-exposure prophylaxis to prevent HIV transmission [13].

Assessments that evaluate patient-reported measures and outcomes (PROs) have the potential to provide a systematic, 
effective, and timely detection of clinically relevant issues by gathering patients' own views and insights before a patientprovider discussion and presenting them in a simple, easily digestible format that can inform action-oriented decisionmaking in a consultation $[4,13,14]$. A review of 27 oncology studies concluded that well-implemented PROs in the clinical setting had a positive impact on detecting otherwise unrecognized problems, improved the monitoring of treatment responses, and enhanced patient-provider communication and patient satisfaction [14]. In the field of HIV, a small number of studies have shown that the use of PROs in care enhances communication between patients and providers and improves providers' ability to detect and monitor symptoms and health behaviors that might otherwise be missed, particularly mental health issues and substance use $[6,15$, 16]. In a large US cohort of HIV clinics, tablet-based PROs have been well accepted and valued by providers [17] and reported as highly acceptable and easy to use by PWH [18].

The use of PROs in the field of HIV care to date has primarily been restricted to clinical trials, research studies, and large academic centers. Despite the clear benefits of successful PRO implementation within clinical care, the effective integration of PROs into care settings for PWH outside of large, highly resourced sites is uncommon. The PROgress study was conducted at 2 community care clinics in North America and aimed to identify the essential program elements that can support the sustainable implementation of PROs into routine HIV care in these settings and to examine the added value of implementing PROs for salient stakeholders, including PWH, providers, and other clinic staff.

\section{Methods}

\section{Study Design}

The PROgress study was designed and monitored with the support of a steering committee, which included PWH, HIV care providers, clinic directors, and healthcare researchers. The design of the study was informed by a targeted literature review focused on the impact of administering PROs within HIV clinical care [13, 19], which evolved throughout the project duration and is available on the PROgress study website at https://progresshivcare.org/.

The study was a prospective, hybrid type three implementation-effectiveness study conducted with 2 community care outpatient clinics with comparable resources: one within $\mathrm{St}$ Michael's Hospital (SMH), Toronto, Ontario, Canada; the other, the Midway Specialty Care Center (MSCC) in Fort Pierce, Florida, USA, between August 2018 and July 2020. The study sites were selected on the basis of several factors, including the number of providers to experience the intervention, caseload, infrastructure, on-site referral resources (e.g., case management, pharmacy, psychiatry), and PRO interest. The study aimed to gain implementation and effectiveness insights regarding the establishment and use of PROs in the clinics, drawing significantly upon an implementation science framework covering 5 outcome domains: reach, effectiveness, adoption, implementation, and maintenance (RE-AIM) [20]. RE-AIM is an established and widely used evaluation framework for assessing the feasibility, quality, and impact of a health intervention [21]. Acceptability was also included as a focus of this research as this was considered a key outcome of interest relating to future scale-up. The primary objectives of the study (Table 1) were (1) to understand and assess the number, proportion, and representativeness of individuals who are willing and able to successfully engage in the process (reach); (2) to assess the impact of the PRO intervention on the patient-provider interaction, clinic operation, and clinical/medical practice (effectiveness); (3) to assess the number, proportion, and representativeness of clinic personnel adopting the intervention (adoption); (4) to assess the degree to which the fidelity of PRO integration is upheld, including consistency of delivery, use as intended, and the time and cost of the intervention (implementation); (5) to assess the extent to which the intervention can be sustained over the longer term at the setting and individual levels (maintenance); and (6) to assess the acceptability of the intervention from the perspective of the stakeholders (patients, providers, and other clinic staff).

The study was divided into three phases for each site: the scoping and preparation phase (preparation), the PRO setup and testing phase (setup), and the PRO delivery phase (delivery). The preparation phase included PRO selection, planning patient-flow maps, provider/clinic staff training, and outcome summary sheet design. The setup phase included setup and testing of PROs. During this phase, eligible PWH self-administered an electronic PRO assessment on-site immediately before a routine care visit, but the assessment results were not shared with the provider. The delivery phase included integration of the PRO intervention within routine care. During this phase, the results of PRO assessments completed by participating PWH were summarized in an instant succinct report that was hand delivered to the provider before seeing the patient. Therefore, patient-provider consultations during the setup phase were carried out without prior PRO feedback, and consultations during the delivery phase were carried out with prior PRO feedback.

\section{Participating PWH}

The study included adults (aged $\geq 18$ years at study entry) with a diagnosis of HIV infection who were attending one of the participating clinics for a routine visit during the study period and who could speak and understand English, Spanish, and/or Haitian Creole sufficiently to be able to complete 
Table 1 Primary objectives and data sources for the PROgress study

\begin{tabular}{|c|c|}
\hline Objectives & Data sources \\
\hline $\begin{array}{l}\text { Reach } \\
\text { To understand and assess the number, proportion, and representative- } \\
\text { ness of individuals who are willing and able to successfully engage } \\
\text { in the process }\end{array}$ & Numbers of PROs initiated and completed \\
\hline $\begin{array}{l}\text { Effectiveness } \\
\text { To assess the impact of the PRO intervention on the patient-provider } \\
\text { interaction, clinic operation, and clinical/medical practice }\end{array}$ & $\begin{array}{l}\text { Chart reviews, one-on-one interviews }{ }^{\mathrm{a}} \text { and structured surveys with } \\
\text { participants (PWH, providers, and clinic staff) }\end{array}$ \\
\hline $\begin{array}{l}\text { Adoption } \\
\text { To assess the number, proportion, and representativeness of clinic } \\
\text { personnel adopting the intervention }\end{array}$ & $\begin{array}{l}\text { Post-interview structured surveys with participating providers and clinic } \\
\text { staff }\end{array}$ \\
\hline $\begin{array}{l}\text { Implementation }^{\mathrm{b}} \\
\text { To assess the degree to which the fidelity of PRO integration is } \\
\text { upheld, including consistency of delivery, use as intended, and the } \\
\text { time and cost of the intervention }\end{array}$ & $\begin{array}{l}\text { One-on-one interviews }{ }^{\mathrm{a}} \text { and post-interview and post-training structured } \\
\text { surveys with participating providers and clinic staff } \\
\text { Time and cost assessment by designated site coordinators }\end{array}$ \\
\hline $\begin{array}{l}\text { Maintenance } \\
\text { To assess the extent to which the intervention can be sustained over } \\
\text { the longer term at the setting and individual levels }\end{array}$ & One-on-one interviews ${ }^{\mathrm{a}}$ with participating providers and clinic staff \\
\hline $\begin{array}{l}\text { Acceptability } \\
\text { To assess the acceptability of the intervention from the perspective of } \\
\text { the stakeholders (patients, providers, and other clinic staff) }\end{array}$ & $\begin{array}{l}\text { Acceptability E-scale and one-on-one interviews and structured surveys } \\
\text { with participating PWH and providers }\end{array}$ \\
\hline
\end{tabular}

$P W H$ people with HIV, $P R O$ patient-reported outcomes assessment

${ }^{a}$ Qualitative analysis of one-on-one interviews is part of planned future work and is not described here

${ }^{\mathrm{b}} \mathrm{A}$ secondary objective was to obtain feedback from stakeholders on the wider project implementation strategies

the PRO assessment. PWH visiting the clinic to see a provider other than their primary HIV care provider or for a non-routine reason, such as acute injury or illness, were not eligible. Those with known acute or significant prohibitive psychiatric, cognitive, or motor impairment or those who appeared acutely intoxicated were excluded.

The planned sample size was $1800 \mathrm{PWH}$ across the two study sites. This was based on attendance estimations over a time period that would enable robust beta testing and integration of PROs within care and the embedding of a process of sufficient length to enable clinic staff to provide valid feedback on implementation.

\section{Participating Providers and Staff}

Providers and clinic staff at each site were invited to participate in a brief survey regarding utility of and satisfaction with PRO implementation.

\section{Preparation}

To anticipate the barriers and facilitators to the successful integration of the PROs, consideration was given to salient implementation constructs, published literature, and the previous experience of PRO integration by members of the team $[6,16,17,22]$. Implementation strategies were then co-created with the sites, which aligned to the desired study objectives and outcomes (Table 2). The Expert Recommendations for Implementing Change (ERIC) were used to help inform this development [23].

Implementation strategies included readiness and needs assessments, including site visits; engaging and involving stakeholders in the process as partners; changing infrastructure; training of staff; and the development of sitespecific action plans.

The initial stages of the preparation phase of the study included the designation of a site research coordinator and the definition of responsibilities for administering, tracking, and responding to PROs (to ensure all steps of the PRO administration were followed for each patient); the development of the electronic PROs (described in more detail in the next section) and patient-flow maps; and the creation of provider and clinic staff training protocols. Staff received on-site training relating to the integration of the PROs into clinical practice, conducted by three study investigators with expertise in implementing PROs $(\mathrm{H}$ Crane, R Fredericksen, and W Lober). To maintain fidelity of the trainers, all three study investigators were present for trainings at each site, and trainings were conducted using a uniform presentation developed by these investigators. Hardware (tablet computers/iPads) and logistical systems were introduced and tested, and any changes deemed necessary to improve implementation efforts were made. 
Table 2 Implementation strategies derived from reference to the Expert Recommendations for Implementing Change (ERIC) [23]

Implementation strategies Strategy details

Use evaluative and iterative strategies

Assess for readiness and identify barriers and facilitators

Conduct local needs assessment

Develop a site-specific action plan

Adapt and tailor implementation to context

Promote adaptability and integrate the PRO process

within the current workflow and clinic operation

Engaging and involving stakeholders in the process as partners to support implementation

Involve leadership from the outset

Involve clinic staff

Identify and prepare champions

Train and educate stakeholders

Conduct educational meetings

Develop educational materials

Change infrastructure

Provide IT infrastructure to enable PRO integration
At the outset, a site visit assessment will be conducted to observe clinic operation and meet with salient pathway stakeholders to promote engagement, identify concerns and challenges (such as capacity barriers), and explore feasible pathway adaptations that will enable integration

We will understand the process and logistics of current clinic flow and how the process can be implemented; this includes understanding the patient journey, options for timing of PRO data collection, and any logistical considerations (e.g., printer locations if the provider uses a printout of the data)

Ahead of implementation, a site-specific action plan will be created for implementation, including the process/flow and choice of PROs to meet clinic needs

The implemented process will be designed to improve patient management and will consider how this can dovetail and improve the current workflow. For example, how can the implementation support or enhance current workflow, such as adopting timings to suit clinic patterns and gathering data that are needed for both clinical and site needs in a more efficient way and not have a detrimental effect on the fidelity of the intervention

We will seek buy-in from senior stakeholders to ensure that we have full endorsement and commitment to implement

The clinical teams will be involved in the decision-making process regarding the PRO intervention, including the PRO domain content, feedback form design, and levels of risk to flag to minimize the need for incremental iterative changes, which can be time-consuming and cause confusion

A role will be defined to champion this process-this may be a research coordinator or similar

Educational materials and training will be conducted to ensure that stakeholders understand the value and process of the PRO data collection intervention

Integrating the process will involve provision of tablet computers with functionality for data collection, the development of a summary sheet of PRO outcomes, and the provision of tablet computers and printers

$I T$ information technology, $P R O$ patient-reported outcomes assessment

\section{PRO Content and Administration}

PROs were administered using a previously developed PRO platform (http://cprohealth.org) and included a selection of PRO instruments that were agreed upon by each site in conjunction with the study sponsor and the evaluation team from the University of Washington. Common, validated, widely used PRO instruments (e.g., PHQ-9 for depression, AUDIT-C for alcohol use) were proposed to clinic leadership and providers for inclusion. Additional measures were chosen based on individual clinic population needs and/or research interest, for example, SMH included measures on Canadian immigration status, sexual behavior under the influence of substances, and health service utilization. PRO instruments were selected for their ability to provide clinically actionable information that could inform decision-making at the time of the consultation. The PRO instruments selected by the study sites covered several clinical domains including antiretroviral adherence, substance use, depression/suicidal ideation, sexual risk behavior, and intimate partner violence (Table 3). Skip logic was applied to reduce the burden of questions for patients where applicable; thus, the number of questions included in the assessments ranged from 65 to 101 at MSCC and from 51 to 100 at SMH. The PRO assessment was administered in electronic format on a tablet computer with touch-screen entry. This form of data capture minimizes the risk of data entry and transfer errors and is also easier for patients as it does not require the use of a mouse or keyboard. Completed PROs were scored using automated algorithms, based on the PRO instrument developers' instructions, and the results were summarized in a 1-page printed report that was shared with the provider immediately before the clinic visit (Fig. 1) and could be subsequently uploaded or added to the medical record. MSCC scanned the PRO results report into the electronic medical record at the end of each day. SMH did not incorporate PRO results reports into medical records. Eligibility for future follow-up assessments within the PRO platform was set for a minimum of 105 days, to minimize potential clinic burden and/or patient survey fatigue, while still capturing changes in key domains (e.g., depression, ART adherence, substance use) in a timely fashion; results from follow-up assessments are not reported here. 
Table 3 Patient-reported outcomes assessment domains and instruments included in PROgress

\begin{tabular}{lll}
\hline Tool & MSCC & SMH \\
\hline Housing & Yes & Yes \\
Nutrition (Canadian Nutrition Screening Tool) [33] & Yes $^{\text {a }}$ & Yes \\
Depression (PHQ-9) [34, 35] & Yes & Yes \\
Anxiety (HIV symptoms inventory) [36] & Yes & Yes \\
Adherence (VAS) & Yes & Yes \\
Satisfaction with HIV medications (HATQoL) [37] & Yes & Yes \\
Nicotine use & Yes & Yes \\
Alcohol use (AUDIT-C) [38] & Yes & Yes \\
Substance use (ASSIST) [39] & Yes & Yes \\
Gender identity & Yes & Yes \\
Sexual risk behavior (SRBI) [40] & Yes & Yes \\
Sexual orientation & Yes & Yes \\
Intimate partner violence (IPV-4) [41] & Yes & Yes \\
Acceptability E-scale [18] & Yes & Yes \\
Other current healthcare & No & Yes \\
Affordability of medication & No & Yes \\
Sex-enhancing substance use, "Chemsex" & No & Yes \\
\hline
\end{tabular}

ASSIST Alcohol, Smoking and Substance Involvement Screening Test, AUDIT-C Alcohol Use Disorders Identification Test for Consumption, HATQoL HIV/AIDS-Targeted Quality of Life, IPV-4 Intimate Partner Violence-4, MSCC Midway Specialty Care Center, PHQ-9 Patient Health Questionnaire 9, SMH St Michael's Hospital, SRBI Sexual Risk Behavior Inventory, VAS visual analog scale

${ }^{a}$ At MSCC, only the weight loss item was included

\section{Assessments and Outcomes}

Evaluation tools used in the study are summarized in Table 4. To understand and assess the number, proportion, and representativeness of PWH who were willing and able to successfully engage in the process (reach), quantitative data were collected on a screening form and in the PRO platform itself. Data included reasons for not completing the assessment, percentage of PWH progressing through the PRO process, and percentage of PWH requiring or requesting support to complete the PRO process. An incomplete PRO was defined as "not advancing beyond sexual risk behavior items"; these items are approximately $75 \%$ of the way through the assessment.

To determine the frequency of discussion of symptoms and behaviors identified by the PRO and the frequency of referral to other care providers on the basis of PRO information (effectiveness), chart reviews of medical records were carried out for same-day clinic visits for participating PWH who completed a PRO in the setup phase (without delivery of PRO feedback to providers; $N=200,100$ per site) and in the delivery phase (with delivery of PRO feedback to providers; N=396, 199 MSCC, 197 SMH). Chart reviews evaluated discussion and referral of depressive symptoms/ suicidal ideation, anxiety, antiretroviral medication adherence and satisfaction, substance use, alcohol use, intimate partner violence (IPV), sexual risk behavior, and nutrition (measures listed in Table 3).

Perspectives of participating PWH on the acceptability and usability of the PRO platform were evaluated by means of the Acceptability E-scale incorporated into the PRO (Supplementary Appendix 1), which measures 7 components of acceptability using Likert scales [18]. This was supplemented by 200 post-visit structured surveys carried out during the delivery phase to assess patient perception of the impact of PROs on care (100 per site; Supplementary Appendix 1). Data on the time spent on completion of PROs were provided from the PRO platform. Time spent on the informed consent process was not included in the calculation of duration of PRO completion time. Perspectives of participating providers and non-medical clinic staff on the utility and value added of integrating PROs into care were evaluated by means of structured surveys $(n=16)$, including statements for which participants were asked to indicate their level of agreement using Likert scales (Supplementary Appendix 1). Assessment of the quality and usefulness of training during the preparation phase was conducted via a post-training structured survey (Supplementary Appendix 1) administered to staff and providers in attendance $(\mathrm{n}=18)$.

\section{Informed Consent Procedures}

Participating PWH provided written informed consent onsite at the time of their care visit before each study activity. There was no remuneration for completing the PRO. For the post-visit structured survey, patients were paid US $\$ 10$ for their participation. Providers and staff provided written informed consent before all study activities; verbal consent was used for the post-training evaluation. Providers and staff were invited to participate in study activities by an on-site research coordinator and were not remunerated for participation. Approval for research involving human participants was granted for all study activities through the SMH Research Ethics Board and through the University of Washington Institutional Review Board for MSCC.

\section{Statistical Analysis}

Statistical analyses were performed using STATA version 14.2 (StataCorp LLC, College Station, TX). Data presentation is descriptive, with mean and standard deviation for continuous variables and percent distribution for categorical variables. Comparison of the chart reviews relating to phases when providers did and did not receive the PRO results used the Fisher exact test with a significance level of 0.05 . 




https://hiv-pros-test.cirg.washington.edu/provider-report-multisession.php?patientID=100941\&projectID=75

Fig. 1 Example of patient-reported outcomes assessment provider feedback summary (based on a fictional patient). AUDIT-C Alcohol Use Disorders Identification Test for Consumption, $D O B$ date

\section{Results}

\section{Study Population}

A total of 1813 eligible PWH were asked to participate in the study, 1632 (90\%) initiated a PRO assessment, and of those, 1630 (>99\%) completed the assessment (Fig. 2). The Acceptability E-scale was completed by $1102 \mathrm{PWH}$.

Demographic data for PWH included in the chart reviews showed that the study population was reflective of the overall clinic populations, and demographic characteristics of participating PWH in the setup and delivery phases were similar (Table 5). Most participating PWH were men, and approximately half were aged $\geq 50$ years (Table 5 ). Anxiety and depression were commonly reported (34\% and $27 \%$ of participating $\mathrm{PWH}$, respectively), and $7 \%$ of participating PWH reported having suicidal ideation on "more than half the days" or "nearly every day" (SMH) or on "several days," "more than half the days," or "nearly every day" (MSCC) of birth, HATQoL HIV/AIDS-Targeted Quality of Life, IPV intimate partner violence, $M R N$ medical record number, $P H Q-9$ Patient Health Questionnaire 9

over the previous 2 weeks (Table 5). One-quarter (25\%) of PWH included in the chart reviews indicated that they were dissatisfied with their HIV medication, responding "some of the time" or more often on one or both HIV/AIDS-Targeted Quality of Life instrument (HATQoL) items “...taking my [HIV] medicine has been a burden" and "...taking my [HIV] medicine has made it hard to live a normal life" (Table 5). This level of dissatisfaction was consistent with results from the total study population (Fig. 3).

Participating providers taking part in the delivery phase survey included 5 medical doctors, 1 nurse practitioner, 1 physician's assistant, 2 pharmacists, and 2 registered nurses. Five non-medical clinic staff also completed delivery phase surveys. The post-training survey was completed by 18 clinic staff (specific roles were not captured in the survey). 


\section{'Reach'-PWH Willing and Able to Successfully Engage with PROs}

Among 1813 eligible PWH asked to participate, 1632 (90\%) provided informed consent and began the PRO assessment. Reasons for the 181 refusals or failures to begin the assessment included language barriers $(n=68,38 \%)$, literacy barriers $(\mathrm{n}=21,12 \%)$, and vision problems including those related to availability of spectacles $(n=11,6 \%)$. Twenty-two PWH (12\%) felt that the PRO assessment was not needed or was not useful (Supplementary Fig. 1). All but 2 of the participating PWH completed the PRO assessment. The mean time taken to complete the PRO assessment was $9 \mathrm{~min}$ across both sites.

\section{'Effectiveness' of PROs on Patient-Provider Interaction, Clinic Operation, and Clinical/Medical Practice}

\section{Impact of PROs on Clinical/Medical Practice Outcomes}

Delivery of the PRO assessment to the provider in advance of the patient's visit increased the number of complex health and behavioral issues that were identified, recorded, and acted on. Comparison of chart reviews from the preparation phase (without PRO feedback to providers) and the delivery phase (with PRO feedback to providers) showed that when provided with PRO assessment data in advance, providers were significantly more likely to document suicidal ideation $(P=0.002)$ and anxiety $(P<0.001)$ and significantly more likely to refer to mental health services for anxiety $(P=0.008$; Table 6). Other notable increases in provider documentation that did not reach statistical significance, but merit further investigation, include dissatisfaction with antiretroviral medication, depression, and having experienced psychological violence (Table 6). There were no differences between sites in rates of documentation (data not shown).

\section{Providers' Experience of Using PROs and Perception of the Impact of PROs on Their Clinical Practice}

The delivery phase structured surveys with providers indicated that PROs helped illuminate patient needs and issues that are often not observable and/or not adequately addressed in consultations. Among 11 providers, 9 (82\%) either agreed or strongly agreed that the PROs helped them prioritize discussion points with the patient, identified topics that might otherwise not have been brought up, led to more discussions on potentially sensitive topics, and added value to the visit overall (Fig. 4A). Most providers (8/11, 73\%) also found that the PROs made their consultation easier.

\section{Providers' Perceived Impact of the Process Upon Their Consultation Workload and Time}

The PROs were regarded as having a minimal and/or manageable impact upon workload and time for providers. Survey results showed some disagreement on whether PROs saved time during the consultation: 5 (45\%) agreed, $3(27 \%)$ disagreed, and 3 (27\%) neither agreed nor disagreed.

\section{Clinic Staff's Perceptions of the Impact of Integration of PROs into the Clinic Process}

Survey results for the clinic staff showed that all 5 agreed that the PRO assessment added value to patient care (Fig. 4B). Clinic staff disagreed whether the PRO assessment saved them time: $2(40 \%)$ agreed, $2(40 \%)$ neither agreed nor disagreed, and 1 (20\%) disagreed (Fig. 4B). Additional tasks completed by the on-site research coordinators were estimated to require approximately 4 min per patient and included explaining the procedure to patients, peripheral monitoring to determine when the patient had finished, and tablet computer stewardship and sanitization.

\section{Patients' Perceptions of the Value, Experience, and Barriers of Introducing PROs into Their Care Consultation with the Provider}

In the post-visit survey, 164/200 (82\%) PWH agreed or strongly agreed that the PRO assessment made their visit to the clinic better (Fig. 5A). Most PWH also agreed or strongly agreed that the PRO assessment helped them consider overall health $(177 / 200,88 \%)$, recall health concerns to raise $(161 / 200,80 \%)$, discuss topics that might otherwise not have arisen $(152 / 200,76 \%)$, discuss issues difficult to speak frankly about (142/200, 71\%), and decide what to talk about $(134 / 200,67 \%)$. Sixty-five percent (129/198) of PWH surveyed reported that they had discussed the burden of their HIV medication and its impact on their life. Comparison of responses between sites found that patients at MSCC were more likely than patients at $\mathrm{SMH}$ to indicate that PROs helped them decide what to talk about (Fig. 5A).

\section{'Adoption' of PROs by Clinic Personnel}

Early and strong leadership endorsement was established through a pre-study site visit, organizational readiness assessment, and joint development of an iterative action plan. This was followed by preparation work by each 
Table 4 Overview of evaluation tools used in the PROgress study

\begin{tabular}{|c|c|c|c|c|c|c|}
\hline Evaluation tool/method & Study phase & Administered & Participants & $\mathrm{n}$ & Measured & Remuneration \\
\hline PRO training evaluation & Preparation & At conclusion of training & Staff and providers & 18 & $\begin{array}{l}\text { Perceived quality of PRO } \\
\text { training }\end{array}$ & None \\
\hline $\begin{array}{l}\text { Acceptability E-scale } \\
\text { survey }\end{array}$ & Setup and delivery & Within PRO assessment & Patients & 1102 & $\begin{array}{l}\text { Acceptability and usabil- } \\
\text { ity of PRO platform }\end{array}$ & None \\
\hline PIPPI survey & Delivery & On-site, after care visit & Patients & 200 & $\begin{array}{l}\text { Perceived impact of } \\
\text { PROs on patient-pro- } \\
\text { vider communication }\end{array}$ & US $\$ 10$ \\
\hline $\begin{array}{l}\text { Brief clinical/non-clinical } \\
\text { staff post-interview } \\
\text { survey }\end{array}$ & Delivery & $\begin{array}{l}\text { At conclusion of one-on- } \\
\text { one interview }\end{array}$ & Staff and providers & 16 & $\begin{array}{l}\text { Perceived utility of PROs } \\
\text { in practice }\end{array}$ & None \\
\hline Chart review & Setup and delivery & $\begin{array}{l}\text { NA, performed by on-site } \\
\text { research coordinator }\end{array}$ & Patients & 596 & $\begin{array}{l}\text { Incidence of provider } \\
\text { documentation of } \\
\text { issues identified by } \\
\text { PRO and subsequent } \\
\text { referral }\end{array}$ & NA \\
\hline
\end{tabular}

NA not applicable, PIPPI perceived impact of PROs on patient-provider interaction, $P R O$ patient-reported outcomes assessment

Fig. 2 PROgress study design and participants. $P W H$ people with HIV, $P R O$ patient-reported outcomes assessment. ${ }^{a}$ Reasons for not initiating the PRO were language barriers $(n=68)$, felt not needed/useful $(n=22)$, literacy barriers $(n=21)$, not in $\operatorname{mood}(n=13)$, vision problems $(\mathrm{n}=11)$, perceived length $(n=10)$, cognitive problems $(n=7)$, too sick $(n=3)$, tired of it $(n=1)$, other reason $(n=6)$, and no reason given $(n=19) .{ }^{b} 2$ participants did not complete the PRO for unknown reasons. 'Including 1102 completed Acceptability E-scales. ${ }^{\mathrm{d}}$ Qualitative analysis of one-on-one interviews is part of planned future work and is not described here. ${ }^{\text {eSetup }}$ phase $=$ without PRO feedback to providers; delivery phase $=$ with $\mathrm{PRO}$ feedback to providers

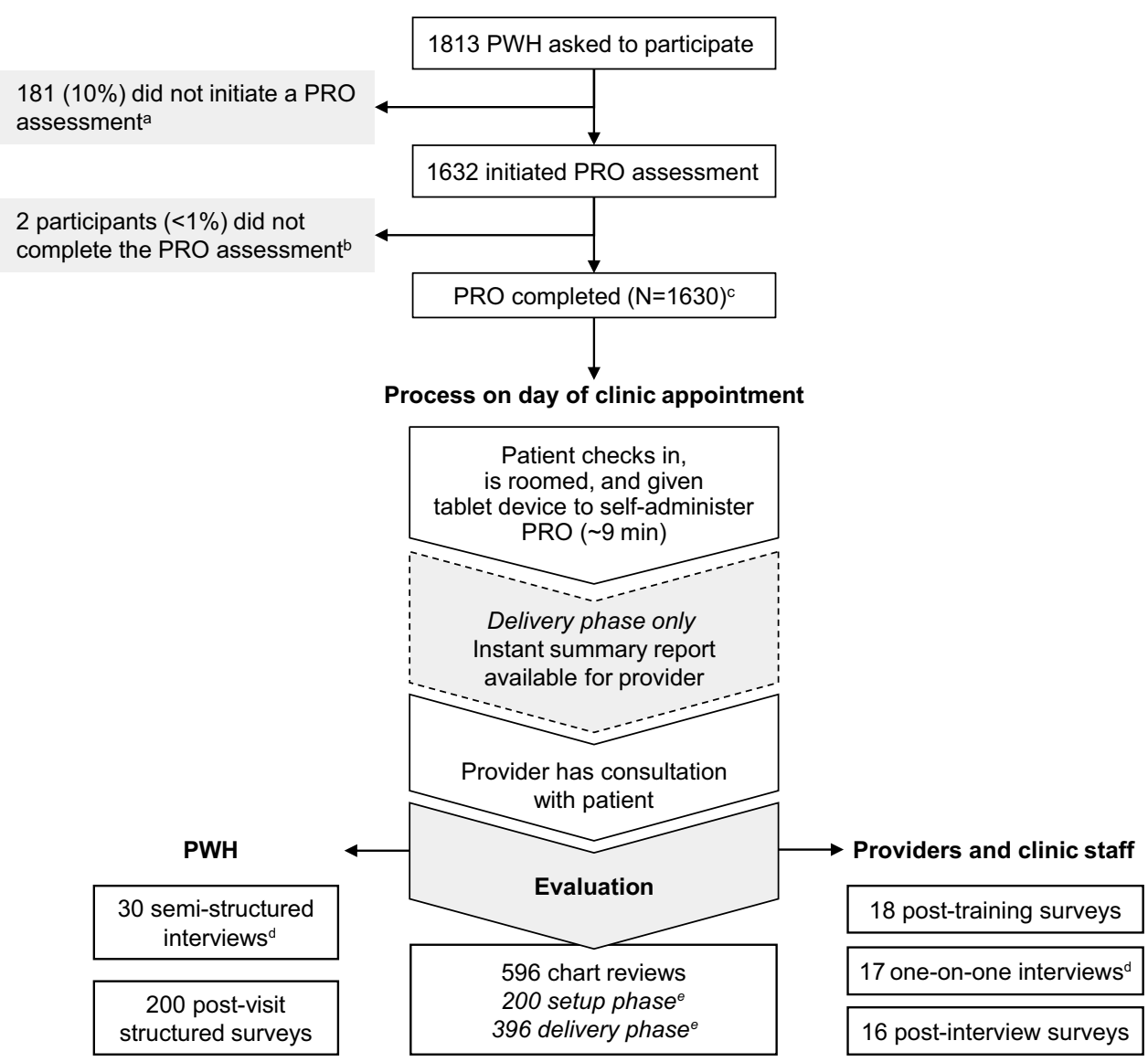

clinic, communicating a process by which the clinic and each role could benefit (e.g., ensuring the capture of information that would already need to be gathered in routine care, thus addressing multiple needs and saving time overall). The PRO assessment was introduced as a hands-on activity for providers and clinic staff during the initial session; this engaged interest and inspired possibilities for application to their needs/setting.

On the basis of the survey findings, there was $100 \%$ adoption among providers and staff of the PRO. 
Table 5 Demographic characteristics, symptoms, and behaviors of participating PWH included in the chart reviews and post-visit structured surveys

\begin{tabular}{|c|c|c|c|c|}
\hline \multirow[t]{2}{*}{ Parameter, n (\%) } & \multicolumn{3}{|l|}{ Chart reviews } & \multirow{2}{*}{$\begin{array}{l}\text { Post-visit } \\
\text { survey } \\
(\mathrm{N}=200)\end{array}$} \\
\hline & $\begin{array}{l}\text { Setup phase } \\
(\mathrm{N}=200)\end{array}$ & $\begin{array}{l}\text { Delivery phase } \\
(\mathrm{N}=396)\end{array}$ & Total $(\mathrm{N}=596)$ & \\
\hline \multicolumn{5}{|l|}{ Age } \\
\hline$<30$ & $24(12)$ & $44(11)$ & $68(11)$ & $16(8)$ \\
\hline 30 to $<40$ & $34(17)$ & $83(21)$ & $117(20)$ & $37(18)$ \\
\hline 40 to $<50$ & $35(18)$ & $74(19)$ & $109(18)$ & $41(20)$ \\
\hline 50 to $<60$ & $50(25)$ & $85(21)$ & $135(23)$ & $50(25)$ \\
\hline$\geq 60$ & $55(28)$ & $110(28)$ & $165(28)$ & $56(28)$ \\
\hline \multicolumn{5}{|l|}{ Sex at birth } \\
\hline Male & $137(68)$ & $272(69)$ & 409 (69) & $144(72)$ \\
\hline Female & $63(32)$ & $124(31)$ & $187(31)$ & $56(28)$ \\
\hline \multicolumn{5}{|l|}{ Race } \\
\hline Black & $83(42)$ & $171(43)$ & $254(43)$ & $81(40)$ \\
\hline White & $79(40)$ & $153(39)$ & $222(37)$ & $76(38)$ \\
\hline Asian & $9(4)$ & $15(4)$ & $24(4)$ & $10(5)$ \\
\hline Other/NS ${ }^{\mathrm{a}}$ & $29(15)$ & $57(14)$ & $86(14)$ & $33(17)$ \\
\hline \multicolumn{5}{|l|}{ Ethnicity } \\
\hline Hispanic & $13(6)$ & $29(7)$ & $42(7)$ & $11(6)$ \\
\hline Symptoms and behaviors & & & & NR \\
\hline Dissatisfaction with $\mathrm{ART}^{\mathrm{b}}$ & $54(27)$ & $97(24)$ & $151(25)$ & \\
\hline ART adherence $\leq 90 \%$ & $36(18)$ & $58(15)$ & $94(16)$ & \\
\hline Any IPV & $25(13)$ & $24(6)$ & $49(8)$ & \\
\hline Suicidal ideation & $13(7)$ & $26(7)$ & $39(7)$ & \\
\hline Anxiety & $62(31)$ & $142(36)$ & $204(34)$ & \\
\hline Depression & $61(31)$ & $99(25)$ & $160(27)$ & \\
\hline Nutrition $(\mathrm{CNST})^{\mathrm{c}}$ & $85(43)$ & $147(37)$ & $232(39)$ & \\
\hline Hazardous drinking (AUDIT-C) & $40(20)$ & $107(27)$ & $147(25)$ & \\
\hline Substance use (ASSIST) & $24(12)$ & $57(14)$ & $81(14)$ & \\
\hline
\end{tabular}

$A R T$ antiretroviral therapy, ASSIST Alcohol, Smoking and Substance Involvement Screening Test, AUDIT$C$ Alcohol Use Disorders Identification Test for Consumption, CNST Canadian Nutrition Screening Tool, HATQoL HIV/AIDS-Targeted Quality of Life, IPV intimate partner violence, $N R$ not recorded, $N S$ not specified, $P W H$ people with HIV, $P R O$ patient-reported outcomes assessment, $R O S$ review of systems

${ }^{a}$ Including aboriginal, First Nations, Middle Eastern, mixed race, and Native American

${ }^{b}$ Responded "some of the time" or greater on one or both of the following HATQoL items: "...taking my [HIV] medicine has been a burden" and "...taking my [HIV] medicine has made it hard to live a normal life"

${ }^{c}$ Weight loss without trying on CNST or ROS item 177 re weight loss, or 176 re weight gain/fat

\section{'Implementation'一The Fidelity of PRO Integration, Including Consistency of Delivery, Use as Intended, and the Time and Cost of the Intervention}

\section{Fidelity of PRO Integration}

Fidelity to core elements was complete and consistent, including training completion at sites by all relevant staff, appointment of a champion/coordinator at sites with defined responsibilities, application of the PRO process to eligible patients according to protocol, and use of a results summary within consultations by providers as part of routine care.

\section{Provider and Staff Satisfaction with Training and Implementation}

All 18 providers and clinic staff who completed the posttraining survey agreed or strongly agreed that the objectives of the training were clearly defined; participation and interaction were encouraged; the topics covered were relevant to them; the content was organized and easy to follow; training objectives were met; time allotted for training was sufficient; and the training increased their confidence in their 
Fig. 3 Patient-reported outcomes assessment responses to HIV/AIDS-Targeted Quality of Life satisfaction with HIV medications questions
In the past 4 weeks, taking my HIV medicine ...has been a burden



All of the time, a lot of the time, some of the time, or a little of the time
None of the time

No answer ability to work with PROs. Most (80\%) clinic staff surveyed agreed that PRO assessments had integrated well into their clinic flow and that PROs were not difficult to implement (Fig. 4B).

\section{Costs of PRO Implementation}

As reported by the on-site research coordinators, the costs of implementing the PRO assessment were low in terms of consumables: the initial purchase of tablet computers/iPads (4 at MSCC and 8 at SMH, at a cost of \$329 each) and printing costs where the assessment report was delivered on paper. The largest costs were human resources, including time for setup, training, monitoring, and reviewing. Once the PRO program was established, the on-site research coordinators estimated that each PRO assessment took around 4 min of time for administrative staff in total, including patient engagement, monitoring, and tablet computer preparation and sanitization. With an average of 7.3 and 10.7 PRO assessments per day at MSCC and $\mathrm{SMH}$, respectively, this equated to $6 \%$ and $9 \%$ of the time of a full-time employee.

\section{'Maintenance'-The Extent to Which the Intervention can be Sustained over the Longer Term}

The future maintenance or sustainability of the PRO assessment process at the study clinics was regarded as having been influenced positively by several factors, including leadership engagement, early stakeholder involvement, planned integration to support and enhance the service, a careful phased introduction, and the demonstration of good value.
There was a desire from both sites to continue with PRO assessments after the end of the study within their own resources.

\section{Acceptability}

Mean scores from patients' responses to the Acceptability E-scale built into the PRO assessment were all above 4, indicating high levels of satisfaction (Fig. 5B). Most participants enjoyed using the assessment (4.09/5) and found it easy to use (4.65/5), well explained (4.56/5), understandable (4.68/5), and helpful in describing their symptoms and health behaviors (4.27/5). They also found the amount of time taken to complete the PRO assessment to be highly acceptable (4.44/5). The overall mean satisfaction rating was 4.39/5. There were site differences in responses to specific questions. Compared with patients at SMH, patients at MSCC were more likely to find the PRO assessment helpful in describing symptoms and behaviors (4.18 vs. 4.38; $P<0.01)$ and were more likely to be satisfied with the assessment overall (4.32 vs. 4.46; $P=0.01$; Fig. 5B).

\section{Discussion}

The PRO assessment evaluated in this study improved the frequency of provider identification, documentation, and referral for complex health and behavioral issues, many of which are known to be less observable, underreported, and/or inadequately addressed in consultations. Differences in documentation between the phases without vs with PRO feedback to providers were significant for anxiety and 
Table 6 Impact of PROs on provider behavior: documentation of symptoms, behaviors, and referrals in the setup phase (without PRO feedback to providers, $\mathrm{N}=200)$ and delivery phase (with PRO feedback to providers, $\mathrm{N}=396$ )

\begin{tabular}{|c|c|c|c|c|c|c|}
\hline \multirow[t]{2}{*}{ Symptom/behavior } & \multirow{2}{*}{$\begin{array}{l}\text { PRO feedback } \\
\text { to providers }{ }^{\mathrm{a}}\end{array}$} & \multirow[t]{2}{*}{$\mathrm{N}^{\mathrm{b}}$} & \multicolumn{2}{|c|}{ Documentation } & \multicolumn{2}{|l|}{ Referral } \\
\hline & & & $\mathrm{n}(\%)^{\mathrm{c}}$ & $P$ value $^{\mathrm{d}}$ & $\overline{\mathrm{n}(\%)^{\mathrm{c}}}$ & $P$ value $^{\mathrm{d}}$ \\
\hline \multirow[t]{2}{*}{ Any IPV } & Without & 25 & $8(32)$ & 0.77 & NA & NA \\
\hline & With & 24 & $9(38)$ & & NA & \\
\hline \multirow[t]{2}{*}{ Sexual risk behavior ${ }^{\mathrm{f}}$} & Without & 14 & $4(29)$ & 1.0 & NA & NA \\
\hline & With & 20 & $7(35)$ & & NA & \\
\hline \multirow[t]{2}{*}{ Adherence $\leq 90 \%$} & Without & 36 & $30(83)$ & 0.77 & NA & NA \\
\hline & With & 58 & $50(86)$ & & NA & \\
\hline \multirow{2}{*}{$\begin{array}{l}\text { Dissatisfaction with ART } \\
\text { regimen }(\mathrm{HATQOL})^{\mathrm{g}}\end{array}$} & Without & 54 & $23(43)$ & 0.09 & NA & NA \\
\hline & With & 97 & $56(58)$ & & NA & \\
\hline \multirow[t]{2}{*}{ Suicidal ideation ${ }^{\mathrm{e}, \mathrm{h}}$} & Without & 13 & $5(38)$ & 0.002 & $7(54)$ & 0.48 \\
\hline & With & 26 & $23(88)$ & & $18(69)$ & \\
\hline \multirow[t]{2}{*}{ Anxiety ${ }^{\mathrm{h}}$} & Without & 62 & $15(24)$ & $<0.001$ & $11(18)$ & 0.008 \\
\hline & With & 142 & $77(54)$ & & $52(37)$ & \\
\hline \multirow[t]{2}{*}{ Depression $^{\mathrm{h}}$} & Without & 61 & $26(43)$ & 0.08 & $19(31)$ & 0.40 \\
\hline & With & 99 & $57(58)$ & & $38(38)$ & \\
\hline \multirow[t]{2}{*}{ Nutrition $(\mathrm{CNST})^{\mathrm{h}, \mathrm{i}}$} & Without & 85 & $31(36)$ & 0.58 & $31(36)$ & 0.58 \\
\hline & With & 147 & $60(41)$ & & $60(41)$ & \\
\hline \multirow[t]{2}{*}{ Hazardous drinking (AUDIT-C) ${ }^{\mathrm{h}}$} & Without & 40 & $12(30)$ & 0.40 & $6(15)$ & 0.38 \\
\hline & With & 107 & $25(23)$ & & $10(9)$ & \\
\hline \multirow[t]{2}{*}{ Substance use (ASSIST) ${ }^{\mathrm{h}}$} & Without & 24 & 7 (29) & 0.80 & $3(12)$ & 1.0 \\
\hline & With & 57 & $20(35)$ & & $7(12)$ & \\
\hline
\end{tabular}

ART antiretroviral therapy, ASSIST Alcohol, Smoking and Substance Involvement Screening Test, AUDIT$C$ Alcohol Use Disorders Identification Test for Consumption, CNST Canadian Nutrition Screening Tool, HATQoL HIV/AIDS-Targeted Quality of Life, IPV intimate partner violence, NA not applicable, PRO patient-reported outcomes assessment, ROS review of systems

${ }^{\text {a }}$ Setup phase $=$ without PRO feedback to providers; delivery phase $=$ with PRO feedback to providers

${ }^{\mathrm{b}}$ Symptom endorsed in PRO assessment

${ }^{c}$ Percent of total endorsed

${ }^{\mathrm{d}}$ Fisher exact test

eProviders were alerted in both phases

${ }^{\mathrm{f}}$ At St Michael's Hospital only

${ }^{g}$ Responded "some of the time" or greater on 1 or both of the following HATQoL items: "...taking my [HIV] medicine has been a burden" and "...taking my [HIV] medicine has made it hard to live a normal life"

${ }^{\mathrm{h}}$ Referrals were counted as discussions

${ }^{\mathrm{i}}$ Weight loss without trying on CNST or ROS item 177 re weight loss, or 176 re weight gain/fat suicidal ideation, both of which were poorly documented during the preparation phase without PRO feedback $(24 \%$ and $38 \%$, respectively) and shifted to being documented in the majority of cases in the delivery phase with PRO feedback. There were also increases in documentation for dissatisfaction with antiretroviral medication, depression, and experience of psychological violence from an intimate partner. For some domains, documentation was infrequent (approximately one-third or fewer cases) during both phases, regardless of PRO feedback, including sexual risk behavior, hazardous drinking, and substance use. This is in contrast with findings from a similar analysis by Crane et al. (2017) in which documentation of 'at-risk alcohol use' and 'substance use' increased after implementing provider feedback of PROs, although the differences were not always significant [16]. The impact on referral (where applicable) in our study was smaller than the impact on documentation, with only patients with anxiety being significantly more frequently referred to mental health services. Differences between domains in terms of documentation and referral may be related to provider perception of the importance of different issues, the provider's comfort with addressing specific issues vs need for additional support, and the availability of appropriate follow-up services. It is notable that approximately one-quarter of participating PWH indicated dissatisfaction with their antiretroviral treatment given that 
Fig. 4 Results of structured surveys carried out during the delivery phase with A providers $(\mathrm{N}=11)$ and $\mathbf{B}$ clinic staff $(\mathrm{N}=5)$ to assess the perceived impact of PROs. PRO patientreported outcomes assessment
A

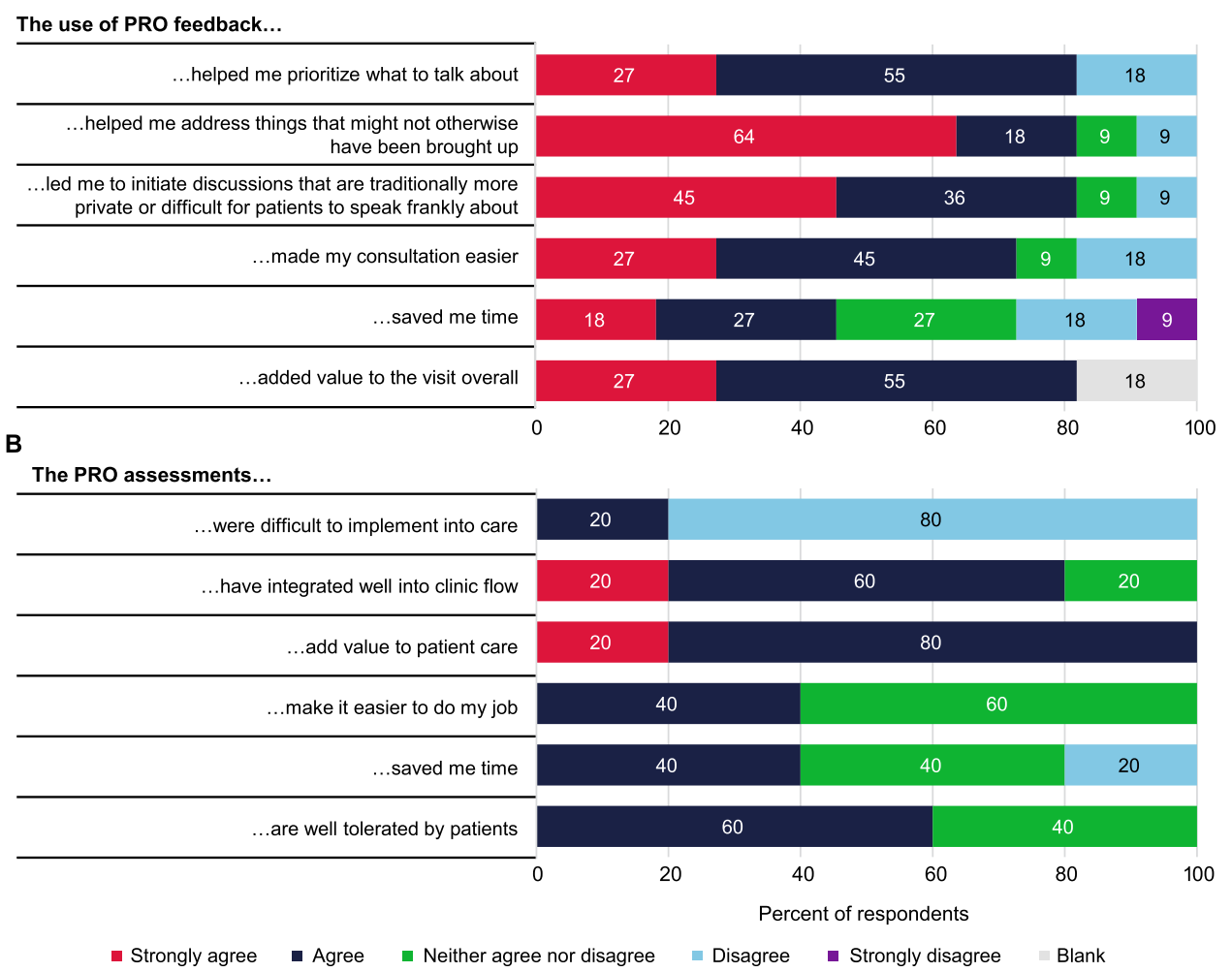

the study was conducted at a time when several once-daily antiretroviral treatment options with low toxicity are available. These results align with data from the Positive Perspectives study, which indicate that many PWH continue to face challenges with their antiretroviral therapy that they may not be raising with their healthcare providers, including difficulties swallowing pills, stress from a daily dosing routine, and a fear of revealing their HIV status by taking pills [24].

The acceptability of the PRO assessment by all stakeholders at the clinics in this study was high and was consistent across all measures employed $[17,25]$. In the survey, most providers agreed that PROs added value to the consultation by helping to prioritize discussion topics and identifying topics that would not otherwise have been addressed. Similarly, survey data from non-medical staff showed a high level of agreement on the ease of integration of PROs into clinic flow and the added value for patients. The Acceptability E-scale built into the PRO assessment showed that PWH also found the PROs useful and easy to use, with a mean overall satisfaction rating of 4.39 out of 5 .

The use of tablet-based PRO assessments was likely an important factor in the high acceptability of PROs in this study. Patients generally prefer tablet-based over paper-based administration [26, 27], and the ability to integrate skip patterns into the design of the assessment (e.g., not asking nonsmokers about smoking patterns) ensures a manageable time burden on patients and decreases impact on patient flow.
Digital administration of PROs has several other advantages, including automated scoring within domains, reducing scoring errors, and the ability to link PRO responses to real-time alerts and to rapidly generate summary results. Additionally, the use of patient portals or URLs has the potential to open access to PROs to a wide range of users, including those with restricted access to a physical clinic. The past decade has seen increasing use of telemedicine in HIV care, and this is likely to be accelerated by the COVID-19 pandemic, which has prompted widespread consideration of more permanent use of remote care delivery methods [28-31]. Challenges to the use of telemedicine in HIV care include concerns over inadequate interpersonal connection between the patient and provider, leading to inability to properly assess complex health issues [28]. To address this concern, it is important to develop strategies that maintain a patientcentered approach to HIV care via telemedicine. The use of digital PROs in this context can reduce reporting bias when in physical healthcare settings, help providers better understand their patients' needs and health behaviors regardless of distance, and identify those most at risk of loss to follow-up.

A language barrier was the most common reason for patients not engaging with the PRO assessment. This could be relatively easily overcome by implementing translations, which for many validated PRO measures are widely available in multiple languages. This was identified early on and was the reason for the addition of a Haitian Creole version 
(A)

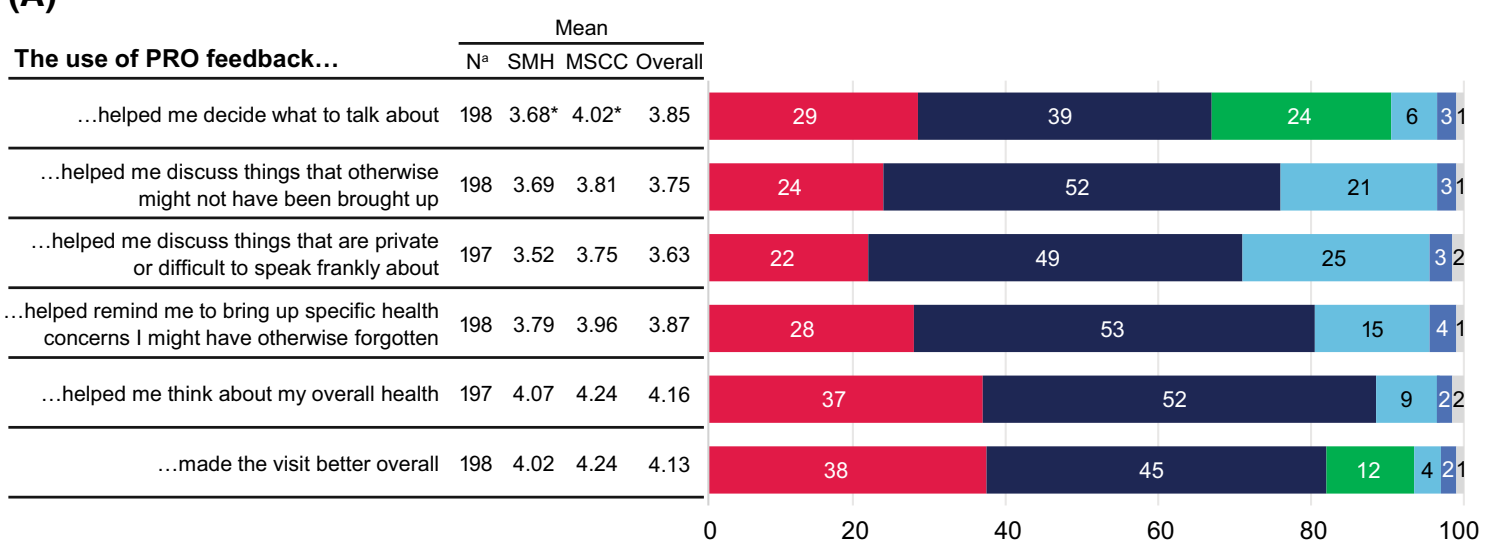

(B)

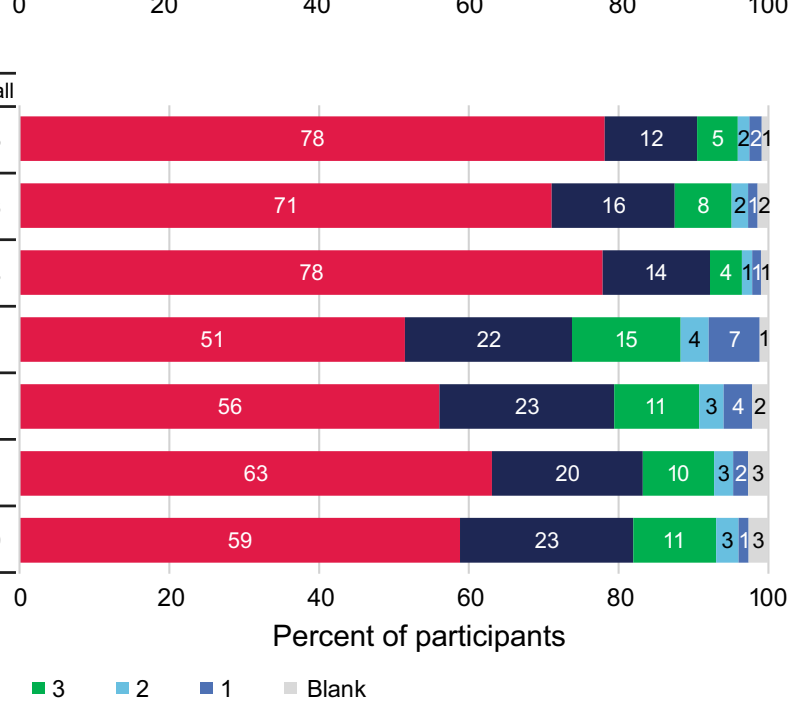

Fig. 5 Results of A PIPPI $(\mathrm{N}=200)$ and B Acceptability E-scale $(\mathrm{N}=1102)$ surveys to assess participants' perceived impact and acceptability of PROs. ${ }^{a}$ Number of participants who responded to the

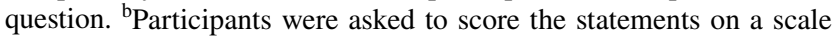
of 1 to 5. For panel A, 1 represented strong disagreement and 5 represented strong agreement. For panel B, 1 represented a low level of

partway through the study, resulting in a decrease in the refusal rates. Using a digital format, there is also the ability to include an audio component for PWH with poor vision or low literacy. A results summary can be generated in the provider's desired language regardless of the language in which the assessment was administered to the patient.

One strength of this study is that it evaluated the implementation of PROs into daily practice in community-based outpatient HIV clinics and therefore may be applicable to a greater number of settings than previously published data from clinical trials or studies conducted at larger, highly resourced academic centers. A further strength is that data were collected from all salient stakeholders, including nonmedical clinic staff for whom the implementation of PROs could have a significant impact. A limitation is that the study was done at only two North American clinics, which were ease, satisfaction, and understanding and 5 represented a high level. *Mean scores significantly different between sites $(P<0.05, t$ test). MSCC Midway Specialty Care Center, PIPPI perceived impact of PROs on patient-provider interaction, $P R O$ patient-reported outcomes assessment, SMH St Michael's Hospital

selected to have characteristics increasing the likelihood of successful implementation of PROs into their practice, such as high leadership engagement for implementation. In addition, since PWH who dislike questionnaires were able to opt out of participating, the study population is likely to be representative of patients who tolerate this type of PRO assessment. Nevertheless, the implementation learnings acquired during the study provide a valuable framework from which to develop customized PRO programs for a variety of situations. These learnings have informed the development of a publicly available PRO implementation toolkit for integrating PRO assessments into routine HIV care (available at https://progresshivcare.org/), which was created as part of this study [32].

While this study provides a rich description of lessons learned from the implementation of a web-based PRO into 
routine HIV care at two outpatient clinics, many questions remain unanswered. Next steps include a focus on the insights of implementation and impact of PROs over time (longitudinal analyses), particularly as it relates to the maintenance phase of RE-AIM and the long-term implementation lessons. As more data accrue, there will be the opportunity to evaluate satisfaction with medications using the HATQoL questions in the context of individual regimens. In addition, as noted in Table 1 and Fig. 2, we have also been conducting provider and patient interviews. This qualitative evaluation will enrich and add context to the quantitative findings presented here. Lastly, the PRO process developed as part of this study is being implemented at a further three sites in the Toronto Ontario HIV Treatment Network in addition to $\mathrm{SMH}$, and there is an intent to extend this to all 21 sites in the network in the future.

In conclusion, in these two North American HIV clinics, providers found PROs with results delivery before patient appointments both useful and acceptable for routine HIV care. PROs may facilitate prioritization of issues to address and expedite identification of sensitive topics, particularly anxiety and suicidal ideation. This may offset additional burden on clinic flow and provider workload. PWH receiving care at the clinics found PROs administered before the appointment to be useful for prioritizing discussion topics with their providers, helping initiate discussion on sensitive issues, and improving comprehensiveness of and satisfaction with care.

Supplementary Information The online version contains supplementary material available at https://doi.org/10.1007/s10461-022-03585-w.

Acknowledgements We thank the patients, providers, and research staff from the two PROgress study clinics, the Midway Specialty Care Center in Fort Pierce, Florida, USA, and St Michael's Hospital in Toronto, Ontario, Canada, for their rich insights and commitment to this work. We thank the wider PROgress study steering committee for their oversight and guidance. This study was funded by ViiV Healthcare. Editorial assistance was provided under the direction of the authors by Esther Race of Race Editorial Ltd and MedThink SciCom, and funded by ViiV Healthcare.

Author Contributions DS, RF, HC, EF, WL, JM, and BJ contributed to the conception of the study. RF, EF, SS, BJ, VH, and DK contributed to the acquisition of data. DS, RF, EF, and SS contributed to the analysis of data. DS, RF, and HC contributed to drafting the manuscript. All authors contributed to the design of the study, the interpretation of data, critically revising the manuscript for important intellectual content, and approve the manuscript for publication.

Funding This study was funded by ViiV Healthcare.

Data Availability Anonymized individual participant data and study documents can be requested for further research from www.clinicalst udydatarequest.com.

Code Availability Not applicable.

\section{Declarations}

Conflict of Interest DS is an employee of ViiV Healthcare and may own stock in GlaxoSmithKline. HC has received grants from ViiV Healthcare. SS has received grants from the Ontario HIV Treatment Network (OHTN). MR has received personal fees from Gilead, ViiV Healthcare, Janssen, and Merck for speaking engagements and/or consultancies. JBe has received personal fees from Merck, ViiV Healthcare, and Gilead for advisory board participation. BJ is an employee of Midway Immunology and Research Center, which was hired by Midway Specialty Care Center to oversee this project. RF, EF, JBa, AM, $\mathrm{KG}, \mathrm{JM}, \mathrm{AK}, \mathrm{VH}, \mathrm{DK}$, and WL have nothing to disclose.

Ethical Approval The study was conducted in full compliance with International Conference on Harmonization Good Clinical Practice Guidelines, the principles of the Declaration of Helsinki, and the laws and regulations of the countries in which the research was conducted. The study was approved by institutional review boards for the participating clinics. Data handling within the PRO system was in accordance with current US privacy and security regulations governing the storage and transmission of protected health information (http://tiny.cc/cirgH IPAApolicies).

Consent to Participate All study participants provided written informed consent.

Consent for Publication Not applicable.

Open Access This article is licensed under a Creative Commons Attribution 4.0 International License, which permits use, sharing, adaptation, distribution and reproduction in any medium or format, as long as you give appropriate credit to the original author(s) and the source, provide a link to the Creative Commons licence, and indicate if changes were made. The images or other third party material in this article are included in the article's Creative Commons licence, unless indicated otherwise in a credit line to the material. If material is not included in the article's Creative Commons licence and your intended use is not permitted by statutory regulation or exceeds the permitted use, you will need to obtain permission directly from the copyright holder. To view a copy of this licence, visit http://creativecommons.org/licenses/by/4.0/.

\section{References}

1. Grinsztejn B, De Castro N, Arnold V, et al. Raltegravir for the treatment of patients co-infected with HIV and tuberculosis (ANRS 12180 Reflate TB): a multicentre, phase 2, noncomparative, open-label, randomised trial. Lancet Infect Dis. 2014;14(6):459-67.

2. Autenrieth CS, Beck EJ, Stelzle D, Mallouris C, Mahy M, Ghys P. Global and regional trends of people living with HIV aged 50 and over: estimates and projections for 2000-2020. PLoS ONE. 2018;13(11):e0207005.

3. Harris TG, Rabkin M, El-Sadr WM. Achieving the fourth 90: healthy aging for people living with HIV. AIDS. 2018;32(12):1563-9.

4. Kall M, Marcellin F, Harding R, Lazarus JV, Carrieri P. Patientreported outcomes to enhance person-centred HIV care. Lancet HIV. 2020;7(1):e59-68.

5. Lazarus JV, Safreed-Harmon K, Barton SE, et al. Beyond viral suppression of HIV - the new quality of life frontier. BMC Med. 2016;14(1):94. 
6. Fredericksen R, Crane PK, Tufano J, et al. Integrating a webbased, patient-administered assessment into primary care for HIVinfected adults. J AIDS HIV Res. 2012;4(2):47-55.

7. Duko B, Ayalew M, Ayano G. The prevalence of alcohol use disorders among people living with HIV/AIDS: a systematic review and meta-analysis. Subst Abuse Treat Prev Policy. 2019;14(1):52.

8. Edelman EJ, Tetrault JM, Fiellin DA. Substance use in older HIVinfected patients. Curr Opin HIV AIDS. 2014;9(4):317-24.

9. Green TC, Kershaw T, Lin H, et al. Patterns of drug use and abuse among aging adults with and without HIV: a latent class analysis of a US Veteran cohort. Drug Alcohol Depend. 2010;110(3):208-20.

10. Do AN, Rosenberg ES, Sullivan PS, et al. Excess burden of depression among HIV-infected persons receiving medical care in the United States: data from the Medical Monitoring Project and the Behavioral Risk Factor Surveillance System. PLoS ONE. 2014;9(3):e92842.

11. Aidala AA, Wilson MG, Shubert V, et al. Housing status, medical care, and health outcomes among people living with HIV/AIDS: a systematic review. Am J Public Health. 2016;106(1):e1-23.

12. Willie TC, Stockman JK, Perler R, Kershaw TS. Associations between intimate partner violence, violence-related policies, and HIV diagnosis rate among women in the United States. Ann Epidemiol. 2018;28(12):881-5.

13. Fredericksen R, Crane HM, Lober W, Fitzsimmons E, Short D. PROgress evidence review and summary: impact of administering patient-reported measures and outcomes (PROs) within HIV routine care. https://progresshivcare.org/assets/PROgress\% 20Review\%20and\%20Summary\%20FINAL\%20Nov2020.pdf. Accessed 1 Nov 2020.

14. Chen J, Ou L, Hollis SJ. A systematic review of the impact of routine collection of patient reported outcome measures on patients, providers and health organisations in an oncologic setting. BMC Health Serv Res. 2013;13:211.

15. Høgh Kølbæk Kjær AS, Rasmussen TA, Hjollund NH, Rodkjaer LO, Storgaard M. Patient-reported outcomes in daily clinical practise in HIV outpatient care. Int J Infect Dis. 2018;69:108-14.

16. Crane HM, Crane PK, Tufano JT, et al. HIV provider documentation and actions following patient reports of at-risk behaviors and conditions when identified by a web-based point-of-care assessment. AIDS Behav. 2017;21(11):3111-21.

17. Fredericksen RJ, Tufano J, Ralston J, et al. Provider perceptions of the value of same-day, electronic patient-reported measures for use in clinical HIV care. AIDS Care. 2016;28(11):1428-33.

18. Fredericksen RJ, Harding BN, Ruderman SA, et al. Patient acceptability and usability of a self-administered electronic patientreported outcome assessment in HIV care: relationship with health behaviors and outcomes. AIDS Care. 2021;33(9):1167-77.

19. Short D, Kotsopoulos N, Crane HM, Fredericksen R. Can patientreported measures and outcomes (PROs) improve routine HIV consultations? A targeted review of the literature. In: Patient Reported Outcome Measures Research Conference, Birmingham, 2018 [abstract 23]

20. Glasgow RE, Harden SM, Gaglio B, et al. RE-AIM planning and evaluation framework: adapting to new science and practice with a 20-year review. Front Public Health. 2019;7:64

21. Glasgow RE, Vogt TM, Boles SM. Evaluating the public health impact of health promotion interventions: the RE-AIM framework. Am J Public Health. 1999;89(9):1322-7.

22. Kitahata MM, Rodriguez B, Haubrich R, et al. Cohort profile: the Centers for AIDS Research Network of Integrated Clinical Systems. Int J Epidemiol. 2008;37(5):948-55.

23. Waltz TJ, Powell BJ, Matthieu MM, et al. Use of concept mapping to characterize relationships among implementation strategies and assess their feasibility and importance: results from the Expert
Recommendations for Implementing Change (ERIC) study. Implement Sci. 2015;10:109.

24. de los Rios P, Okoli C, Castellanos E, et al. Physical, emotional, and psychosocial challenges associated with daily dosing of HIV medications and their impact on indicators of quality of life: findings from the Positive Perspectives study. AIDS Behav. 2021;25(3):961-72.

25. Fredericksen RJ, Short D, Fitzsimmons E, et al. Patient perceptions of the utility and impact of a same-day self-administered routine electronic patient-reported outcomes (PRO) assessment in HIV care in two North American clinics. In 27th Annual Conference of International Society for Quality of Life Research, Virtual, 2020 [abstract 3062].

26. Campbell N, Ali F, Finlay AY, Salek SS. Equivalence of electronic and paper-based patient-reported outcome measures. Qual Life Res. 2015;24(8):1949-61.

27. Fanning J, McAuley E. A comparison of tablet computer and paper-based questionnaires in healthy aging research. JMIR Res Protoc. 2014;3(3):e38.

28. Anderson K, Francis T, Ibanez-Carrasco F, Globerman J. Physician's perceptions of telemedicine in HIV care provision: a cross-sectional web-based survey. JMIR Public Health Surveill. 2017;3(2):e31.

29. Coombes CE, Gregory ME. The current and future use of telemedicine in infectious diseases practice. Curr Infect Dis Rep. 2019;21(11):41.

30. Gras G. Use of telemedicine in the management of infectious diseases. Med Mal Infect. 2018;48(4):231-7.

31. Mgbako O, Miller EH, Santoro AF, et al. COVID-19, telemedicine, and patient empowerment in HIV care and research. AIDS Behav. 2020;24(7):1990-3.

32. Fredericksen RJ, Short D, Fitzsimmons E, et al. PROgress implementation toolkit: integrating patient-reported outcomes (PROs) assessments into routine HIV care. https://progresshivcare.org/ assets/PROgress\%20Implementation\%20Toolkit-FINAL-Nov20 20.pdf. Accessed 2 Jan 2021.

33. Laporte $\mathrm{M}$, Keller $\mathrm{HH}$, Payette $\mathrm{H}$, et al. Validity and reliability of the new Canadian Nutrition Screening Tool in the 'real-world' hospital setting. Eur J Clin Nutr. 2015;69(5):558-64.

34. Kroenke K, Spitzer RL, Williams JB. The PHQ-9: validity of a brief depression severity measure. J Gen Intern Med. 2001;16(9):606-13.

35. Spitzer RL, Kroenke K, Williams JB. Validation and utility of a self-report version of PRIME-MD: the PHQ primary care study. Primary Care Evaluation of Mental Disorders. Patient Health Questionnaire. JAMA. 1999;282(18):1737-44.

36. Justice AC, Holmes W, Gifford AL, et al. Development and validation of a self-completed HIV symptom index. J Clin Epidemiol. 2001;54(suppl 1):S77-90.

37. Holmes WC, Shea JA. A new HIV/AIDS-targeted quality of life (HAT-QoL) instrument: development, reliability, and validity. Med Care. 1998;36(2):138-54.

38. Bush K, Kivlahan DR, McDonell MB, Fihn SD, Bradley KA. The AUDIT alcohol consumption questions (AUDIT-C): an effective brief screening test for problem drinking. Ambulatory Care Quality Improvement Project (ACQUIP). Alcohol Use Disorders Identification Test. Arch Intern Med. 1998;158(16):1789-95.

39. Newcombe DA, Humeniuk RE, Ali R. Validation of the World Health Organization Alcohol, Smoking and Substance Involvement Screening Test (ASSIST): report of results from the Australian site. Drug Alcohol Rev. 2005;24(3):217-26.

40. Fredericksen RJ, Mayer KH, Gibbons LE, et al. Development and content validation of a patient-reported sexual risk measure for use in primary care. J Gen Intern Med. 2018;33(10):1661-8.

41. Fitzsimmons E, Loo S, Dougherty S, et al. Development and content validation of the IPV-4, a brief patient-reported measure of 
intimate partner violence for use in HIV care. In: 26th Annual Conference of International Society for Quality of Life Research, San Diego, 2019 [abstract 1106].
Publisher's Note Springer Nature remains neutral with regard to jurisdictional claims in published maps and institutional affiliations. 